\title{
A assistência multiprofissional a pacientes em tratamento de COVID-19 e a minimização do distanciamento familiar em um serviço de pronto atendimento em Manaus, Amazonas
}

\author{
Multi-professional assistance to patients undergoing COVID-19 treatment and \\ minimizing family distance in emergency service in Manaus, Amazonas
}

La asistencia multiprofesional a los pacientes en tratamiento de COVID-19 y la minimización de la distancia familiar en un servicio de atención inmediata en Manaus, Amazonas

Alyne Batista Martins ${ }^{1 *}$, Luciane Coimbra Schmidt ${ }^{1}$, Maria Jelcilene Marques de Sousa Lima ${ }^{1}$, Lídia Dias dos Santos ${ }^{1}$, Orácio Carvalho Ribeiro Junior ${ }^{1}$.

\section{RESUMO}

Objetivo: Relatar a assistência multiprofissional aos pacientes em tratamento de COVID-19 com vistas à minimização do distanciamento familiar em um serviço de pronto atendimento em Manaus. Relato de Experiência: Quando foi declarada a transmissão comunitária no estado do Amazonas, em abril de 2020, todas as unidades da rede de alta complexidade estadual foram designadas para a pandemia passaram a receber pacientes com o diagnóstico, o que gerou lotação de leitos, atrasos de transferências e sobrecarregou todas as unidades de urgência e emergência. O Serviço de Pronto Atendimento estava tratando os pacientes suspeitos e confirmados para Covid-19 com os suprimentos e recursos humanos que possuía. A situação trazida pela Covid-19 fez repensar práticas profissionais, de forma que se realizasse um cuidado mais humanizado aos usuários e familiares. O acolhimento da família, vídeo-chamadas entre pacientes e famílias mediadas pela equipe multiprofissional e outras medidas foram estabelecidas para fortalecer o enfrentamento à doença. Considerações Finais: As medidas realizadas foram de grande importância para atenuar os impactos do distanciamento familiar dos pacientes e enfrentamento à doença, assim como o conforto aos familiares que podiam visualizar, mesmo que virtualmente, o cuidado que estava sendo oferecido.

Palavras-chave: Equipe de assistência ao paciente, Humanização da assistência, Infecções por coronavírus.

\begin{abstract}
Objective: To report multi-professional assistance to patients undergoing COVID-19 treatment to minimize family distance in an emergency care service in Manaus. Experience Report: When community transmission was, declared in the state of Amazonas, in April 2020, all units of the state's high complexity network were designated for the pandemic and started receiving patients with the diagnosis, which generated bed capacity, delays transfers and overloaded all urgency and emergency units. The Emergency Department was treating suspected and confirmed patients for Covid-19 with the supplies and human resources it had. The situation brought about by Covid-19 made rethinking professional practices so that more humanized care was provided to users and family members. The reception of the family, video calls between patients and families mediated by the multidisciplinary team, and other measures were established to strengthen the coping with the disease. Final Considerations: The measures taken were of great importance to mitigate the impacts of patients' family distancing and coping with the disease, as well as the comfort for family members who could see, even if virtually, the care being offered.
\end{abstract}

Keywords: Patient care team, Humanization of assistance, Coronavirus infections.

${ }^{1}$ Secretaria de Saúde do Estado do Amazonas (SUSAM), Manaus-AM. *E-mail: alynebatista19@gmail.com 


\section{RESUMEN}

Objetivo: Reportar la asistencia multiprofesional a los pacientes en tratamiento de COVID-19 con vistas a minimizar la distancia familiar en un servicio de atención inmediata en Manaus. Informe de Experiencia: Cuando se declaró la transmisión comunitaria en el estado de Amazonas, en abril de 2020, todas las unidades de la red de alta complejidad estatal fueron designadas para la pandemia comenzaron a recibir pacientes con el diagnóstico, lo que generó la capacidad de camas, retrasos en los traslados y sobrecargó todas las unidades de emergencia. El Servicio de Atención Inmediata estaba tratando a los pacientes sospechosos y confirmados para Covid-19 con los suministros y recursos humanos que poseía. La situación traída por Covid19 hizo repensar prácticas profesionales, de forma que se realizara un cuidado más humanizado a los usuarios y familiares. La acogida de la familia, videollamadas entre pacientes y familias mediadas por el equipo multiprofesional y otras medidas fueron establecidas para fortalecer el enfrentamiento a la enfermedad. Consideraciones Finales: Las medidas realizadas fueron de gran importancia para atenuar los impactos del distanciamiento familiar de los pacientes y enfrentamiento a la enfermedad, así como el confort a los familiares que podían visualizar, aunque virtualmente, el cuidado que estaba siendo ofrecido.

Palabras-Clave: Grupo de atención al paciente, Humanización de la atención, Infecciones por coronavirus.

\section{INTRODUÇÃO}

Em novembro de 2019, na cidade de Wuhan, na China, foi detectado um surto de doença respiratória causado pelo novo coronavírus (SARS-CoV-2), sendo esta denominada posteriormente como Covid-19. A doença foi declarada pela Organização Mundial da Saúde (OMS), em 30 de janeiro de 2020, como uma

Emergência de Saúde Pública de Importância Internacional - o nível mais alto de alerta - e, em menos de 2 meses, em 11 de março de 2020, tornou-se uma pandemia (BRASIL MS, 2020a; ORGANIZAÇÃO PANAMERICANA DA SAÚDE, 2020a).

A Covid-19 apresenta amplo espectro clínico podendo os portadores serem assintomáticos, oligossintomáticos ou manifestarem quadros graves como a Síndrome Respiratória Aguda Grave (SRAG), especialmente em grupos de risco como idosos, gestantes, imunodeprimidos e com diversas comorbidades.

A transmissão do vírus SARS-CoV-2 acontece por meio de gotículas de saliva ou secreção nasal, de pessoa a pessoa, após tosse, fala ou espirro, e ainda de forma indireta por meio de objetos e superfícies contaminadas (BRASIL MS, 2020b).

Entre os sinais e sintomas clínicos da doença, destacam-se: febre, tosse seca, fadiga, produção de escarro, dispneia, dor de garganta, cefaleia, mialgia ou artralgia, calafrios, náuseas e vômitos, congestão nasal, diarreia, hemoptise, e congestão conjuntival. Os estudos observam que cerca de $80 \%$ dos portadores do vírus desenvolvem sintomas considerados de leves a moderados, com ou sem a presença de pneumonia.

Por estes pontos, faz-se necessária a rápida identificação da infecção para que a implementação da terapêutica possa acontecer de maneira oportuna, além do que, o isolamento dos sujeitos infectados é fundamental para a contenção da doença e se enquadra nos moldes das emergências em saúde pública (BERNHEIM A, et al., 2020; GUAN W, et al., 2020; LIMA, 2020).

Por essa razão, dentre as medidas não farmacológicas para prevenção e controle da transmissão do coronavírus, é recomendado o distanciamento social, a higienização das mãos, etiqueta respiratória e o uso de máscaras tanto no ambiente intra quanto no extra-hospitalar.

Sendo que, no ambiente intra-hospitalar, desde a triagem, o paciente segue um fluxo diferenciado para atendimento sendo isolado dos demais pacientes que não são suspeitos para Covid-19 e, se houver internação, segue em isolamento em enfermaria, podendo ficar com outros do mesmo diagnóstico, porém sem o acompanhamento de familiares e visitantes (BRASIL MS, 2020c; ORGANIZAÇÃO PAN-AMERICANA DA SAÚDE, 2020a; CENTERS FOR DISEASE CONTROL AND PREVENTION, 2020a). 
Neste contexto, o isolamento social quer no contexto comunitário, quer no contexto hospitalar, constituise como ferramenta essencial a nível global para a contenção da pandemia, bem como, para a redução da vulnerabilidade dos sujeitos nos mais variados contextos e deve ser permeada por intenso processo educativo sobre sua importância, para que ocorra adesão por parte da população (FARIAS HS, 2020).

Segundo Ornell F, et al. (2020) afirmam que as incertezas que a doença desconhecida traz em relação as suas características e a uma terapia comprovadamente eficaz, até então, têm consequências e implicações diretas no cotidiano e saúde mental da população.

Algumas ações de ajuda psicossocial e de saúde mental ao enfrentamento da pandemia foram publicadas pela OMS e o Centro de Controle e Prevenção de Doenças dos Estados Unidos (CDC) (ORGANIZAÇÃO PAN-AMERICANA DA SAÚDE, 2020b; CENTERS FOR DISEASE CONTROL AND PREVENTION, 2020b).

Por outro lado, apesar de serem fundamentais para a contenção da pandemia, os processos que geram o isolamento no âmbito individual e/ou coletivo, pode desencadear no sujeito diferentes tipos de mal-estar, como: representação de ser impotente, tédio, solidão, tristeza, irritabilidade e diversos tipos de medo, relacionados principalmente com o morrer em função dos danos causados pela infecção. Estas alterações podem levar a consequências como privação do sono e perde de apetite, fatos que não corroboram para a recuperação da saúde do sujeito, mas, tende a agravá-la (LIMA RC, 2020b).

O paciente que se encontra distante da sua família, seja no isolamento do tratamento domiciliar, mas, especialmente, na internação nas unidades de saúde pode apresentar-se extremamente vulnerável. É necessário que medidas sejam implementadas para atenuar impactos psicossomáticos, estados disfóricos, risco de depressão e suicídio e piora na evolução da doença (SHIGEMURA J, et al., 2020).

Neste sentindo, é necessário que as práticas de cuidados a estes sujeitos possam dar-se de maneira ampla, levando em consideração o ambiente onde os mesmos estão inseridos, suas relações familiares e sociais, para que o impacto da doença primária não afete outros componentes da saúde desses sujeitos (SCHMIDT B, 2020). Assim, como o atendimento multiprofissional pode minimizar o distanciamento familiar destes pacientes e fortalecer o enfrentamento à doença?

Neste contexto, o objetivo deste trabalho é relatar a assistência multiprofissional aos pacientes em tratamento de COVID-19 com vistas à minimização do distanciamento familiar em um serviço de pronto atendimento em Manaus - AM.

\section{RELATO DA EXPERIÊNCIA}

O Serviço de Pronto Atendimento (SPA) em Manaus é uma unidade de saúde atende demandas clínicas e cirúrgicas emergenciais de baixas complexidades e, durante a pandemia em Manaus, atendeu também pacientes suspeitos de Covid-19. Com o período de pico da pandemia no estado, as unidades da rede de alta complexidade designadas para este evento estavam sobrecarregadas e, consequentemente, as unidades de urgência e emergência também estavam em estafa.

Por essa razão, o SPA estava tratando os pacientes suspeitos e confirmados para o novo coronavírus com os suprimentos e recursos humanos que possuía. Rotineiramente, pacientes em período de internação recebem visitas e possuem acompanhantes, porém, como conhecida a alta transmissibilidade do vírus SARSCoV-2, esta rotina, dentre tantas outras, foi modificada.

A situação atípica para muitas relações de saúde-doença trazida pela Covid-19 também fez repensar práticas profissionais de forma que se realizasse um cuidado mais humanizado aos usuários e familiares. Compreende-se que o desenvolvimento da humanização perpassa pelo acolhimento das pessoas que estão em adoecimento, dos familiares fragilizados pela doença e pelo distanciamento, onde os seus entes encontram-se internados em unidades restritas para a assistência à saúde com medidas impostas para redução dos riscos de contaminação. Desta forma, a equipe multiprofissional buscou criar alternativas para minimizar os impactos desse distanciamento. 
O acolhimento dos familiares pelo Serviço Social tornou-se uma ferramenta muito importante para repassar as informações acerca do quadro de saúde, solicitação de materiais e utensílios e até alimentos de predileção do paciente para o incentivo do apetite destes, assim como contatos de referência familiar, orientação sobre possíveis transferências hospitalares com suporte adequado para realização de exames e continuidade do tratamento.

Além disso, em comunhão com os profissionais da Enfermagem e médicos assistenciais, foram permitidas e realizadas vídeo-chamadas e ligações entre os pacientes e familiares, ou ainda, repassados aos pacientes vídeos e áudios enviados para eles. Antes dessas medidas, familiares aglomeravam-se na área externa da unidade em busca de notícias.

Então, foram combinados horários para as ligações a fim de que estivessem em maior número possível e contatar com o paciente internado no intuito de transmitir palavras de encorajamento para aqueles que se encontravam vulneráveis, além de minimizar o esforço que o simples ato de conversar trazia aos que estavam com intensa dispneia.

$\mathrm{Na}$ cultura amazônica, tradicionalmente, usam-se remédios caseiros empiricamente para tratar diversas comorbidades. Em consonância com médicos plantonistas e em respeito à fé alheia, foram permitidos chás e remédios caseiros a alguns pacientes que faziam questão desse tratamento e aguardavam leitos para transferência sem previsão alguma.

Também foi aplicada medida não farmacológica de forma multiprofissional como banho de sol com suporte de oxigênio, monitorização de oximetria de pulso e cadeira de rodas para mudança de ambiente, a fim de proporcionar um conforto e visualização da paisagem no estacionamento da unidade, onde em uma situação parecida, conseguiu-se realizar o encontro presencial de um membro da família com um paciente idoso que estava internado há mais de 20 dias na instituição e sem visitas presenciais de familiares.

Alinhado ao Serviço Social da unidade e equipe de Enfermagem e médica, e seguindo os protocolos de paramentação de Equipamentos de Proteção Individual para o familiar e distanciamento, o referido paciente idoso pôde se despedir do filho, pois após três dias o mesmo evoluiu a óbito na unidade. Percebeu-se que após estas pequenas medidas os pacientes mostravam-se mais calmos e otimistas. Além da aceitação para possíveis transferências hospitalares visando à melhora do seu quadro clínico.

A partir do contexto das ações desenvolvidas, percebe-se o esforço da equipe em ir além dos cuidados técnicos próprios para a situação clínicas dos usuários, mas, um cuidado que se preocupou com a extensão daqueles indivíduos, suas famílias, suas crenças, considerando de fato o conceito ampliado de saúde e pondo em prática os princípios e diretrizes da política nacional de humanização, em um contexto assistencial desafiador.

\section{DISCUSSÃO}

O Boletim Epidemiológico disponibilizado pela Fundação de Vigilância em Saúde do Amazonas registrou no dia 13 de março de 2020 o primeiro caso confirmado de Covid-19 no estado, no final do mesmo mês constavam 80 casos confirmados, sendo $92,5 \%$ na capital Manaus, já o primeiro óbito foi confirmado no dia 24 do corrente mês.

Em pouco mais de um mês, precisamente no dia 16 de abril, o estado apresentava 1.719 casos confirmados já distribuídos entre 22 municípios do Amazonas, uma incidência de 41,5 casos por 100.000 habitantes, e uma marca de 124 óbitos. Desenvolveu a forma grave da doença, Síndrome Respiratória Aguda Grave (SRAG), 340 casos até então (FUNDAÇÃO DE VIGILÂNCIA EM SAÚDE, 2020).

Em menos de um mês, os jornais já noticiavam que o Amazonas estava na lista de transição para aceleração descontrolada do coronavírus, do Ministério da Saúde, ao mesmo tempo em que o hospital de referência para Covid-19 atingia a capacidade máxima operacional necessitando de mais profissionais, assim como a corrida do governo para ampliação de leitos (DINIZ C, 2020). Diante de tal cenário, onde houve uma explosão de casos e a falta de estrutura hospitalar referenciada, é plausível a reação amedrontada da população frente a uma doença que apresentava uma transmissão assustadora, assim como a lotação das demais unidades hospitalares. 
Com a mudança de práticas e rotinas hospitalares a comunicação tornou-se prejudicada entre os pacientes e suas famílias. Visto que as visitas têm uma grande importância como apoio psicológico aos fragilizados, corroboram as intervenções realizadas pela equipe de enfermagem e serviço social CRISPIM D, et al (2020) quando sugerem que pacientes não sejam mantidos sem contato com o meio externo visto que existem diversas possibilidades de aproximação virtual por meios de aplicativos como o whatsapp, que é considerado pelo Conselho Federal de Medicina (CFM) uma ferramenta de comunicação entre médicos e pacientes, conforme parecer ํำ14/2017, e na atual conjuntura foi usado para aproximação com a família.

Sugerem ainda um "time de comunicação e acolhimento aos familiares" que não seja composto por profissionais que estejam prestando cuidados diretos, indicam-se psicólogos e assistentes sociais. Estes devem alinhar junto à família os fluxos de comunicação, horários pré-estabelecidos e orientação a um familiar responsável para reunir os demais, com ao menos uma intervenção diária e duração de 5 a 10 minutos de vídeo-chamadas para aqueles pacientes que possuem comunicação verbal efetiva e com áudios ou chamadas por viva-voz para os que não possuem condições verbais (CRISPIM D, et al., 2020).

Infelizmente no ambiente de trabalho referido não constavam profissionais de Psicologia. Notou-se também que poucos estudos ou relatos de experiência estão relacionados com o impacto da pandemia Covid19 para a comunicação com os pacientes ou descrevendo as emoções destes que estão hospitalizados. Em contrapartida são inúmeros os que relacionam a doença e o conflito emocional enfrentado pelos profissionais da saúde, o que também é de grande relevância.

As ações voltadas à minimização do distanciamento familiar dos pacientes internados fazem-se fundamentais para a não deterioração da saúde psíquica e emocional destes. A experiência de outros contextos epidêmicos mostra que os sujeitos nesta condição clínica temem ser alvos de abandono familiar e dos amigos, além dos preconceitos sociais associados à sua condição de portador da doença, sendo necessária as ações que mitiguem tais impactos (SCHMIDT B, 2020).

Segundo a Política Nacional de Humanização (PNH), humanizar é uma inclusão de processos de gestão e cuidados, associando gestores, profissionais da saúde, usuários e suas redes sociofamiliares nos processos de cuidado, estimulando novas formas de cuidar e de organizar o trabalho.

$\mathrm{Na}$ PNH usuários e trabalhadores devem reconhecer o papel de cada um para produzir saúde, ampliar autonomia e responsabilidades, isto define um dos princípios que regem a política: o protagonismo e corresponsabilidade e autonomia dos sujeitos e coletivos, (BRASIL MS, 2013).

Este princípio foi bastante evidente durante nossa experiência com os pacientes acometidos por Covid-19 em suas hospitalizações quando foi construído o acolhimento de forma coletiva, o respeito à fé alheia, medidas para atenuar o distanciamento familiar e fortalecimento na relação entre equipe, usuário e família.

Concluiu-se que as medidas realizadas através das mudanças das rotinas da unidade foram de grande importância para atenuar os impactos do distanciamento familiar dos pacientes e enfrentamento à doença, assim como o conforto aos familiares que podiam visualizar, mesmo que virtualmente, o cuidado que estava sendo oferecido. Além do que mostrou à equipe uma nova forma de trabalhar frente às situações caóticas que uma pandemia pode gerar.

Observou-se a diminuição de sintomas de ansiedade, tristeza e fragilidade dos pacientes e estabeleceu maior confiança na equipe multiprofissional durante sua assistência e cuidado quando houve o estreitamento das relações entre os atores participantes deste processo.

A Humanização no Serviço de Pronto Atendimento no contexto da pandemia do Covid-19 se deu de forma acolhedora, buscando contemplar a integralidade dos sujeitos no processo de cuidado, promoção, reabilitação e recuperação da saúde. Além do apoio mútuo dos profissionais de saúde da assistência, visando o encorajamento, esperança e fé de cada um.

Espera-se que os profissionais de saúde também tenham um melhor preparo para enfrentamento de situações caóticas. Assim como outros estudos sejam divulgados elencando as medidas usadas para aliviar a situação do distanciamento no ambiente intra-hospitalar. 


\section{REFERÊNCIAS}

1. BERNHEIM A, et al. Chest CT Findings in Coronavirus Disease 2019 (COVID-19): Relationship to Duration of Infection. Radiology, 2020; 295(3): 685-691.

2. BRASIL. Ministério da Saúde. Secretaria de Vigilância em Saúde. Centro de Operações de Emergência -COE. Guia de Vigilância Epidemiológica para Infecção Humana pela COVID-19. Emergência de Saúde Pública Nacional pela Doença pelo Coronavírus 2019. Vigilância Integrada de Síndromes Respiratórias Agudas Doença pelo Coronavírus 2019, Influenza e outros vírus respiratórios/ COE/SVS/MS. Abr, 2020b.

3. BRASIL. Ministério da Saúde. Secretaria de Vigilância em Saúde. Centro de Operações de Emergência -COE. Boletim Epidemiológico. Infecção Humana pelo novo Coronavírus (2019 -nCov) COE/SVS/MS. n. 2. Fev, 2020c.

4. BRASIL. Ministério da Saúde. Secretaria de Atenção à Saúde. Política Nacional de Humanização. Rede HumanizaSUS. Brasília, DF: 1aㅡ edição - 1aㅡ reimpressão, 2013.

5. BRASIL. Ministério da Saúde. Secretaria de Atenção Especializada à Saúde. Departamento de Atenção Hospitalar, Domiciliar e de Urgência. Protocolo de manejo clínico da Covid-19 na Atenção Especializada [recurso eletrônico] / Ministério da Saúde, Secretaria de Atenção Especializada à Saúde, Departamento de Atenção Hospitalar, Domiciliar e de Urgência. - 1. ed. rev. - Brasília : Ministério da Saúde, 2020a.

6. CENTERS FOR DISEASE CONTROL AND PREVENTION. Interim Infection Prevention and Control Recommendations for Patients with Suspected or Confirmed Coronavírus Disease 2019 (COVID-19) in Healthcare Settings. 2020a. [Atualizado em Jul 9, 2020].

7. CENTERS FOR DISEASE CONTROL AND PREVENTION. Mental health and coping during COVID-19. Atlanta: CDC; 2020 b.

8. CONSELHO FEDERAL DE MEDICINA. Parecer ํำ14/2017. Assunto: Uso do Whatsapp em ambiente hospitalar, 2017.

9. CRISPIM D, et al. Comunicação difícil e COVID-19. Recomendações práticas para comunicação e acolhimento em diferentes cenários da pandemia. Associação Médica de Minas Gerais, 2017.

10. DINIZ C. Há um mês, Amazonas registrava primeiro caso de Covid-19; Número passa de 1,2 mil, com mais de 60 mortes. G1 Amazonas, 2020.

11. FARIAS HS. O avanço da Covid-19 e o isolamento social como estratégia para redução da vulnerabilidade. Espaço e Economia. Revista Brasileira de Geografia Econômica, 2020; 17: 1-13.

12. FUNDAÇÃO DE VIGILÂNCIA EM SAÚDE. Estado do Amazonas. Situação Epidemiológica de COVID-19 e da Síndrome Respiratória Aguda Grave no Estado do Amazonas, 2020.

13. GUAN W, et al. Clinical Characteristics of Coronavirus Disease 2019 in China. New England Journal Of Medicine, 2020; 382(18): 1708-1720.

14. LIMA CMAO. Information about the new coronavirus disease (COVID-19). Radiologia Brasileira, 2020a; 53(2): V-VI.

15. LIMA RC. Distanciamento e isolamento sociais pela Covid-19 no Brasil: impactos na saúde mental. Physis: Revista de Saúde Coletiva, 2020b: 30(2): 1-10.

16. ORGANIZAÇÃO PAN-AMERICANA DA SAÚDE. Considerações psicossociais e de saúde mental durante o surto de COVID-19, 18 de março de 2020b.

17. ORGANIZAÇÃO PAN-AMERICANA DA SAÚDE. Folha informativa - COVID-19 (doença causada pelo novo coronavírus) [Internet]. Brasília: Organização Pan-Americana da Saúde; 2020a.

18. ORNELL F, et al. "Pandemic fear" and COVID-19: mental health burden and strategies. Braz J Psychiatry, 2020; 42(3):232-235.

19. SCHMIDT B, et al. Saúde mental e intervenções psicológicas diante da pandemia do novo coronavírus (COVID-19). Estudos de Psicologia, 2020; 37: 1-13.

20. SHIGEMURA J, et al. Public responses to the novel 2019 coronavírus $(2019-n C o V)$ in Japan: Mental health consequences and target populations. Psychiatry and Clinical Neurosciences, 2020; 74: 277-283. 